\title{
Sparse Exponential Family Principal Component Analysis
}

\author{
Meng Lu ${ }^{\mathrm{a}}$, Jianhua Z. Huang ${ }^{\mathrm{b}}$, Xiaoning Qian ${ }^{\mathrm{a}, *}$ \\ ${ }^{a}$ Department of Electrical and Computer Engineering, Texas A\&MM University, College \\ Station, TX, US, 77840. \\ ${ }^{b}$ Department of Statistics, Texas A\&SM University, College Station, TX, US, 77840.
}

\begin{abstract}
We propose a Sparse exponential family Principal Component Analysis (SePCA) method suitable for any type of data following exponential family distributions, to achieve simultaneous dimension reduction and variable selection for better interpretation of the results. Because of the generality of exponential family distributions, the method can be applied to a wide range of applications, in particular when analyzing high dimensional next-generation sequencing data and genetic mutation data in genomics. The use of sparsity-inducing penalty helps produce sparse principal component loading vectors such that the principal components can focus on informative variables. By using an equivalent dual form of the formulated optimization problem for SePCA, we derive optimal solutions with efficient iterative closed-form updating rules. The results from both simulation experiments and real-world applications have demonstrated the superiority of our SePCA in reconstruction accuracy and computational efficiency over traditional exponential family PCA (ePCA), the existing Sparse PCA (SPCA) and Sparse Logistic PCA (SLPCA) algorithms.
\end{abstract}

Keywords: dimension reduction; sparsity; exponential family principal component analysis.

\footnotetext{
* Corresponding author

Email address: xqian@ece.tamu.edu (Xiaoning Qian)
}

Preprint submitted to Pattern Recognition

May 8, 2016

(C) 2016. This manuscript version is made available under the Elsevier user license http://www.elsevier.com/open-access/userlicense/1.0/ 


\section{Introduction}

Dimension reduction methods are widely used for many data analytic applications such as computer vision, data mining, and bioinformatics. In addition to deriving low dimensional projections for model complexity reduction and re5 producibility of learning results, people often would like to know the physical meanings of the original variables and how they contribute to these projections. For example, when analyzing images, it is of much interest to know which image regions are crucial to represent or capture the essential information contained in the given images. Identifying variables expressing the maximum data variation will also be of much interest for next-generation sequencing data analysis since it would help greatly reduce the profiling cost for biomarker discovery. To achieve these goals in diverse real-world applications, one faces two critical challenges: how to handle diverse data types arising from different applications and how to obtain meaningful interpretation of analysis results. Exponential family PCA

15 (ePCA) methods [1, 2, 3] and Sparse PCA (SPCA) methods [4, 5, 6, 7] are well-known to address these two issues separately. However, to the best of our knowledge, it seems that no one has proposed a method to address these two issues simultaneously. In this article, we propose a Sparse exponential family PCA (SePCA) method for dimension reduction with both the capability of addressing the interpretation issue and the generality of applications to any type of data following exponential family distributions.

For probabilistic interpretation, ePCA aims to find the low-dimensional projections of a set of canonical parameters that maximize the likelihood of the observed data. PCA is a special case of ePCA by assuming that the conditional probability of each data point given its corresponding canonical parametersmean vectors - follows a Gaussian distribution. By extending the Gaussian distribution of the conditional probability to other members of the exponential family, ePCA naturally generalizes PCA to be more suitable to handle various data types, including binary and count data for example, other than continuous data that is often assumed to follow a Gaussian distribution. To achieve 
better performance for dimension reduction, an appropriate assumption of the distribution for a given certain data type is desirable. For example, Bernoulli distribution is appropriate for binary data; multinomial distribution for categorical data; Poisson distribution for count data. This generalization of PCA is analogous to the extension of ordinary linear regression models to generalized linear models (GLM) 8. However, ePCA still suffers from the interpretation issue of PCA, which motivates us to derive SePCA by introducing sparsity regularization to the loading vectors. The sparsity could be achieved by adding a regularization term on loading vectors to the objective function of ePCA, which 40 is similar to the way of formulating sparsity regularized GLMs.

Finding an efficient algorithm to solve SePCA is challenging due to a couple of mixed difficulties from SPCA and ePCA from the involved non-convex constraints and the non-convex complex objective function of multiple variables. It has always been challenging to solve either ePCA or SPCA, not to mention 45 solving them simultaneously in SePCA. Several approaches have been proposed to solve either ePCA or SPCA [1, 3, 4, 5, 6, 9, 10, A generic algorithm directly optimizes the objective function of ePCA [1, 2]; however, it is inefficient to deal with non-quadratic and complex likelihood functions for many exponential family members. An alternative effective way is to optimize an auxiliary function of the log-likelihood to achieve the solutions by approximating the objective function with its lower bound. Majorization-Minimization (MM) algorithm [11, 12] has been applied to efficiently solve Sparse Logistic PCA (SLPCA) for binary data with closed-form updating rules [9]. However, it is hard to find appropriate auxiliary functions that can lead to efficient closed-form updating rules for other members of the exponential family such as multinomial distribution and Poisson distribution. Recently, Guo [3] has proposed an efficient algorithm to solve ePCA by transforming the regularized primal problem to an equivalent dual problem with the optimal solution found at the stationary point. Following this idea, we transform the SePCA problem to a dual problem, in which the objec${ }_{60}$ tive function with respect to the principal components (PCs) and the principal component loading vectors has a similar form as the sparse PCA problem for- 
mulated by Shen and Huang (sPCA_rSVD) [6]. We can then solve the SePCA problem by alternately updating unknown variables using efficient closed-form updating rules that lead to favorable computational efficiency.

The rest of the paper is organized as follows. Section 2 briefly reviews classical PCA in a probabilistic modeling framework, from which it could be naturally extended to ePCA. We also introduce the SPCA problem and the algorithm for solving it at the end of this section. Section 3 describes the formulation of SePCA, with an efficient alternative updating algorithm to solve it. The computational complexity analysis of the solution algorithm is also provided. Section 4 illustrates the performance of SePCA compared with Zou's SPCA [5] and a previous SLPCA method [9] via the experiments on both simulated and real-world data. Section 5 concludes the paper and discusses our future directions.

\section{Related Work}

In this section, we review relevant concepts and probabilistic models that form the foundations of SePCA. We introduce PCA from a probabilistic modeling perspective and naturally extend it to the exponential family. From this point of view, PCA is formulated as a Maximum-Likelihood Estimation (MLE) so problem, which estimates the low-dimensional projections of a set of canonical parameters by assuming that the conditional probability of each data point given its canonical parameters follows a Gaussian distribution [13]. Similarly, the ePCA tailored to some other types of data could also be modeled as such a MLE problem by assuming that the conditional probability follows a corre85 sponding distribution in the exponential family other than Gaussian. To give a flavor of SePCA, we introduce SPCA as a simple case and discuss an efficient strategy to solve it at the end of this section.

\subsection{Principal Component Analysis}

Given a set of samples $\boldsymbol{x}_{1}, \ldots, \boldsymbol{x}_{N} \in \mathbb{R}^{D}$, PCA projects the data into a 90 principal-component subspace with a lower dimension $L(\leq D)$ and meanwhile 
attempts to preserve the maximum data variation. An alternative interpretation of PCA from a probabilistic perspective assumes that the data points are approximated by linear projections of low-dimensional latent variables plus a Gaussian noise. For each sample $\boldsymbol{x}_{n}(1 \leq n \leq N)$, given its corresponding 95 vector of latent variables $\boldsymbol{z}_{n}$ that lies in the principal-component subspace, we assume

$$
\boldsymbol{x}_{n}=W^{T} \boldsymbol{z}_{n}+\boldsymbol{b}+\boldsymbol{\epsilon},
$$

where $W$ is a principal loading matrix whose rows span the principal-component subspace; $\boldsymbol{b}$ is a bias vector and $\boldsymbol{\epsilon}$ follows a Gaussian distribution $N\left(0, \sigma^{2} I\right)$. Assuming a vector of canonical parameters $\boldsymbol{\theta}_{n}=W^{T} \boldsymbol{z}_{n}+\boldsymbol{b}$, the conditional probability of $\boldsymbol{x}_{n}$ given $\boldsymbol{\theta}_{n}$ is then represented as:

$$
p\left(\boldsymbol{x}_{n} \mid \boldsymbol{\theta}_{n}\right) \sim N\left(\boldsymbol{x}_{n} \mid \boldsymbol{\theta}_{n}, \sigma^{2} I\right)
$$

and the conditional probability of $\boldsymbol{x}_{n}$ given $\boldsymbol{z}_{n}$ is:

$$
p\left(\boldsymbol{x}_{n} \mid \boldsymbol{z}_{n}\right) \sim N\left(\boldsymbol{x}_{n} \mid W^{T} \boldsymbol{z}_{n}+\boldsymbol{b}, \sigma^{2} I\right) .
$$

PCA is then formulated as an optimization problem of maximizing the loglikelihood of the data set with respect to $\boldsymbol{z}_{n}, W$, and $\boldsymbol{b}$, where the objective function is:

$$
\sum_{n}-\left\|\boldsymbol{x}_{n}-\left(W^{T} \boldsymbol{z}_{n}+\boldsymbol{b}\right)\right\|^{2} \quad \text { s.t. } \quad W W^{T}=I
$$
of Euclidean distances from data points to their projections in the principalcomponent subspace, which is exactly the other interpretation of PCA [14].

\subsection{Exponential Family PCA}

From a probabilistic perspective, it is natural to generalize PCA to the exponential family. In the exponential family, a probabilistic latent variable model representing the conditional distribution of a data sample $\boldsymbol{x}_{n}$ has such a general form [1]:

$$
p\left(\boldsymbol{x}_{n} \mid \boldsymbol{\theta}_{n}\right)=\exp \left(\boldsymbol{\theta}_{n}^{T} \boldsymbol{x}_{n}+\log q\left(\boldsymbol{x}_{n}\right)-A\left(\boldsymbol{\theta}_{n}\right)\right),
$$


where $\boldsymbol{\theta}_{n}$ denotes the corresponding canonical parameters corresponding to the sample $\boldsymbol{x}_{n} \cdot A\left(\boldsymbol{x}_{n}\right)$ is the log-normalization factor with the form based on the base measure $q\left(\boldsymbol{x}_{n}\right): \log \int \exp \left(\boldsymbol{\theta}_{n}^{T} \boldsymbol{x}_{n}\right) q\left(\boldsymbol{x}_{n}\right) d \boldsymbol{x}_{n}$, which ensures that the sum of the conditional probabilities over the domain of $\boldsymbol{x}_{n}$ equals 1 . The probability distribution functions for the members in the exponential family are mainly differentiated by the form of $A(\cdot)$ function. Consequently, the data log-likelihood with respect to the canonical parameters may be of a quadratic form (for Gaussian) or not (for others). Taking Gaussian for instance, $A\left(\boldsymbol{\theta}_{n}\right)$ takes a form of $\boldsymbol{\theta}_{n}^{2} / 2$ to ensure a Gaussian distribution function. Then, its data log-likelihood function given $\boldsymbol{\theta}$ is equivalent to

$$
\sum_{n}-\left\|\boldsymbol{x}_{n}-\boldsymbol{\theta}_{n}\right\|^{2}
$$

up to a constant. The canonical parameters $\boldsymbol{\theta}_{n}$ are further parameterized with a form of $W^{T} \boldsymbol{z}_{n}+\boldsymbol{b}$ using lower-dimensional latent variables $\boldsymbol{z}_{n}$, principal loading matrix $W$ and a bias vector $\boldsymbol{b}$ for dimension reduction. After substituting $\boldsymbol{\theta}_{n}$ into (3), we arrive at (1), which is exactly the objective function of PCA derived by MLE.

In general, ePCA can be achieved by maximizing the generalized likelihood based on a general form of the probability function shown in (2). After substituting $\boldsymbol{\theta}_{n}$ by $\boldsymbol{z}_{n}, W$, and $\boldsymbol{b}$, ePCA is then formulated as the following problem:

$$
\min _{Z, \boldsymbol{b}} \min _{W: W W^{T}=I} \sum_{n} A\left(W^{T} \boldsymbol{z}_{n}+\boldsymbol{b}\right)-\operatorname{tr}\left(\left(Z W+\mathbf{1} \boldsymbol{b}^{T}\right) X^{T}\right),
$$

where $Z$ is the $N \times L$ principal component score matrix whose $n$-th row is $\boldsymbol{z}_{n}$. A probabilistic graphical model to illustrate ePCA is shown in Figure1. Note that the principal component subspace is derived for canonical parameters instead of sample data directly.

Similar to PCA, ePCA encounters the interpretation issue for high dimensional data - a principal component that involves too many variables is hard to interpret. This motivates us to develop a method that can produce sparse PC loading vectors for ePCA. 


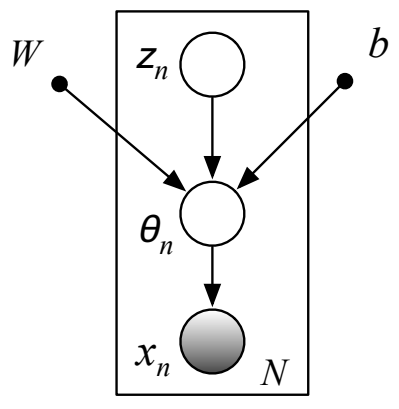

Figure 1: Probabilistic graphical model for ePCA.

\subsection{Sparse $P C A$}

Before discussing SePCA, we briefly review SPCA, which is a special case of SePCA with the Gaussian distribution assumption, and has been studied by numerous papers [5, 6, 7, 15]. Assuming that the data set has been centralized with zero-mean across samples, an intuitive formulation of the SPCA problem is as follows:

$$
\min _{Z} \min _{W: W W^{T}=I}\|X-Z W\|^{2}+\sum_{l=1}^{L} P\left(W_{l}\right)
$$

with the aim to achieve dimension reduction using only a set of significantly contributing original variables, where $P(\cdot)$ is a general convex penalty function that imposes sparsity on $W_{l}$, the $l$-th row of the loading matrix $W$. A major difficulty in solving this problem is caused by the simultaneously imposed orthonormal constraints and the sparse penalty on the loading vectors.

Zou et al. [5] treat SPCA as a penalized regression problem using an elasticnet penalty $P\left(W_{l}\right)=\lambda_{0}|| W_{l} \|_{F}^{2}+\lambda_{l}\left|W_{l}\right|_{1}$ and attempt to minimize the regression error when regressing the PCs on the original variables. They propose a "selfcontained" regression approach to decouple the orthonormal constraints and the elastic-net penalty, which then turns the problem into the following penalized regression problem:

$$
\min _{A: A^{T} A=I} \min _{W}\left\|X-X W^{T} A^{T}\right\|^{2}+\lambda_{0}\|W\|_{F}^{2}+\sum_{l=1}^{L} \lambda_{l}\left|W_{l}\right| .
$$


When $A$ is fixed, the minimization of $W_{l}$ is equivalent to solving an elastic-net regression problem:

$$
\min _{W_{l}}|| X A_{l}-X W_{l}^{T}\left\|^{2}+\lambda_{0}\right\| W_{l} \|_{F}^{2}+\lambda_{l}\left|W_{l}\right| .
$$

Alternatively, we can also decouple the constraints by reformulating (5) as

$$
\min _{Z: Z^{T} Z=I} \min _{W}\|X-Z W\|^{2}+\sum_{l=1}^{L} P\left(W_{l}\right),
$$

which is equivalent to sPCA-rSVD [] when $l=1$.

Problems (6) and (7) are closely related with similar forms in both constraints and objective functions. Although their objective functions are not jointly convex and the constraints are non-convex, both of them can be solved using the same strategy by alternately minimizing variables using closed-form updating rules. When $A$ or $Z$ is fixed, the minimization of $W_{l}$ is a penalized regression problem. When $W$ is fixed, $A$ or $Z$ can be updated according to Theorem 1 proposed in [5] and [16] given below:

Theorem 1. Reduced-Rank Procrustes Rotation. Given two matrices $M_{N \times D}$ and $N_{N \times L}$, consider the constrained minimization problem

$$
\min _{A}\left\|M-N A^{T}\right\|^{2} \quad \text { s.t. } \quad A^{T} A=I_{L \times L} .
$$

Suppose that the Singular Value Decomposition (SVD) of $M^{T} N$ is in the form of $U D V^{T}$, then $\hat{A}=U V^{T}$.

When $A$ is initialized by the first $L$ right eigenvectors of $X$, the above strategy solving the problem (6) can be illustrated as a two-stage procedure: first PCA is performed on $X$; and then sparse approximations are estimated for the loading vectors. This approach leads to efficient solutions demonstrated by the experimental results in [5]. Analogously, when $Z$ is initialized by the first $L$ left eigenvectors of $X$, the above strategy solving the problem (7) also acts as the same two-stage analysis and is expected to lead to efficient solutions. We will introduce such a similar strategy to help solve SePCA and investigate its efficiency in the following sections. 


\section{Sparse Exponential Family PCA (SePCA)}

\subsection{Problem formulation}

We extend the SPCA problem (7) to the exponential family by combining it with (4) and formulate the SePCA problem as follows:

$$
\min _{Z: Z^{T} Z=I} \min _{W, \boldsymbol{b}} \sum_{n} A\left(W^{T} \boldsymbol{z}_{n}+\boldsymbol{b}\right)-\operatorname{tr}\left(\left(Z W+\mathbf{1 b}^{T}\right) X^{T}\right)+P(W, \boldsymbol{b}),
$$

where $P(W, \boldsymbol{b})$ is the penalty term that equals $\lambda_{0}\left\|Z W+\mathbf{1 b}^{T}\right\|^{2}+\sum_{l=1}^{L} \lambda_{l}\left|W_{l}\right|$. The $l_{2}$-norm penalty term is involved here to ensure the stable reconstruction of principal components when $N<D$ and $X$ is not a full rank matrix. It could also be interpreted as a Gaussian prior for canonical parameters to ensure the stability of the model. The tuning parameter $\lambda_{l}$ controls the sparsity of loading vectors. A larger value of $\lambda_{l}$ will cause fewer non-zero elements in $W_{l}$, which are explicitly interpreted as the variables contributing to the $l$-th principal component. SePCA will reduce to ePCA when $\lambda_{0}$ and $\lambda_{l}$ are equal to 0 .

This penalized maximum-likelihood estimator attempts to estimate the optimal loading vectors that are sparse and meanwhile maintain the minimum reconstruction error. Only the variables corresponding to the non-zero elements in a loading vector are selected to construct the corresponding principal component.

\subsection{Reformulation of the objective function}

Based on the observation that for general exponential family distributions, the objective function can be complex (not a quadratic function as in classical PCA) and is not jointly convex on $Z$ and $W$, it is difficult and unsatisfactory to directly solve (8) by alternately updating the unknown variables. Instead, we transform this problem into an equivalent problem by its conjugate dual that can be solved more effectively and efficiently.

The reformulation is achieved via replacing the term $A\left(W^{T} \boldsymbol{z}_{n}\right)$, which is not jointly convex in $Z$ and $W$, by introducing its convex conjugate. The convex conjugate for a function $h(\boldsymbol{\alpha})$ is defined as:

$$
h^{*}(\boldsymbol{u})=\sup _{\boldsymbol{\alpha} \in M}<\boldsymbol{u}, \boldsymbol{\alpha}>-h(\boldsymbol{\alpha})
$$


where $h^{*}(\boldsymbol{u})$ is always convex since the maximum of a linear function is convex. Let $A^{*}(\cdot)$ denotes the convex conjugate of $A(\cdot)$. The explicit form of $A(\cdot)$ and $A^{*}(\cdot)$ specific to a distribution in the exponential family are discussed in [17.

Let $\Theta$ be a $N \times D$ matrix whose $n$-th row is $\boldsymbol{\theta}_{n}$. We first rewrite 8 by introducing linear constraints as follows:

$$
\begin{aligned}
\min _{Z: Z^{T} Z=I} \min _{W, \boldsymbol{b}} \min _{\Theta} & \sum_{n} A\left(\boldsymbol{\theta}_{n}\right)+g(Z, W, \boldsymbol{b}) \\
\text { s.t. } & \boldsymbol{\theta}_{n}=W^{T} \boldsymbol{z}_{n}+\boldsymbol{b}, \quad 1 \leq n \leq N,
\end{aligned}
$$

where $g(Z, W, \boldsymbol{b})=-\operatorname{tr}\left(\left(Z W+\mathbf{1 b}^{T}\right) X^{T}\right)+\Omega(W, \boldsymbol{b})$. Due to the complex form of function $A(\cdot)$, it is difficult to directly solve (9). We first transform the minimization of $A(\cdot)$ to its equivalent dual problem to replace the complex $A(\cdot)$ based on the following Lemma 1:

Lemma 1. Let $U$ be the $N \times D$ matrix whose $n$-th row is $\boldsymbol{u}_{n}$. The inner minimization of (9) with respect to $\Theta$ is equivalent to solving the dual problem:

$$
\max _{U}-\sum_{n} A^{*}\left(-\boldsymbol{u}_{n}\right)-<\boldsymbol{u}_{n}, W^{T} \boldsymbol{z}_{n}+\boldsymbol{b}>+g(Z, W, \boldsymbol{b}) .
$$

Proof. The Lagrangian of (9) is defined as

$$
\sum_{n} A\left(\boldsymbol{\theta}_{n}\right)+<\boldsymbol{u}_{n},\left(\boldsymbol{\theta}_{n}-W^{T} \boldsymbol{z}_{n}-\boldsymbol{b}\right)>+g(Z, W, \boldsymbol{b}) .
$$

The inner minimization of (9) on $\Theta$ can be reformulated as the saddle point problem

$$
\min _{\Theta} \max _{U} \sum_{n} A\left(\boldsymbol{\theta}_{n}\right)+<\boldsymbol{u}_{n}, \boldsymbol{\theta}_{n}>-<\boldsymbol{u}_{n}, W^{T} \boldsymbol{z}_{n}+\boldsymbol{b}>+g(Z, W, \boldsymbol{b}) .
$$

Since the inner minimization of (9) on $\Theta$ is a convex problem with feasible linear constraints, it satisfies Slater's conditions for strong duality and the order of minimization and maximization in 10 can be exchanged:

$$
\begin{aligned}
& \max _{U} \min _{\Theta} \sum_{n} A\left(\boldsymbol{\theta}_{n}\right)+<\boldsymbol{u}_{n}, \boldsymbol{\theta}_{n}>-<\boldsymbol{u}_{n}, W^{T} \boldsymbol{z}_{n}+\boldsymbol{b}>+g(Z, W, \boldsymbol{b}) \\
\equiv & \max _{U}-\left(\max _{\Theta} \sum_{n}-A\left(\boldsymbol{\theta}_{n}\right)-<\boldsymbol{u}_{n}, \boldsymbol{\theta}_{n}>\right)-<\boldsymbol{u}_{n}, W^{T} \boldsymbol{z}_{n}+\boldsymbol{b}>+g(Z, W, \boldsymbol{b}) \\
\equiv & \max _{U}-\sum_{n} A^{*}\left(-\boldsymbol{u}_{n}\right)-<\boldsymbol{u}_{n}, W^{T} \boldsymbol{z}_{n}+\boldsymbol{b}>+g(Z, W, \boldsymbol{b}),
\end{aligned}
$$


which completes the proof for the lemma.

Then, based on Lemma 1, the original optimization problem (8) can be transformed to an equivalent dual problem by Theorem 2 :

Theorem 2. The optimization problem (8) is equivalent to

$$
\min _{Z: Z^{T} Z=I} \min _{W, \boldsymbol{b}} \max _{U}-\sum_{n} A^{*}\left(-\boldsymbol{u}_{n}\right)-\operatorname{tr}\left(\left(Z W+\mathbf{1} \boldsymbol{b}^{T}\right)(U+X)^{T}\right)+\Omega(W, \boldsymbol{b}) .
$$

Proof. It suffices to show that (9) is equivalent to (11). From Lemma 1, it is straightforward to prove that $(9)$ is equivalent to its dual problem:

$$
\begin{aligned}
& \min _{Z: Z^{T} Z=I} \min _{W, \boldsymbol{b}} \max _{U}-\sum_{n} A^{*}\left(-\boldsymbol{u}_{n}\right)-<\boldsymbol{u}_{n}, W^{T} \boldsymbol{z}_{n}+\boldsymbol{b}>+g(Z, W, \boldsymbol{b}) \\
\equiv & \min _{Z: Z^{T} Z=I} \min _{W, \boldsymbol{b}} \max _{U}-\sum_{n} A^{*}\left(-\boldsymbol{u}_{n}\right)-\operatorname{tr}\left(\left(Z W+\mathbf{1} \boldsymbol{b}^{T}\right)(U+X)^{T}\right)+\Omega(W, \boldsymbol{b}),
\end{aligned}
$$

which leads to (11) and completes the proof for the theorem.

We will then focus on solving the equivalent dual problem 11 in the following subsection.

\subsection{Closed-form updating rules}

Despite of the non-quadratic objective function and non-convex constraints, we can still find closed-form updating rules to solve (11) with good solution quality. The algorithm based on these updating rules will converge much faster than first-order iterative updating approaches. The solutions are achieved by alternately updating the unknown variables based on the closed-form solutions, which are given below.

Let $f(Z, W, \boldsymbol{b}, U)$ denote the objective function of this min-max problem (11). Obviously, $f(\cdot, \cdot, \cdot, U)$ is concave in $U$. In each iteration, we can update $U$ by solving the following optimization problem:

$$
\max _{U}-\sum_{n} A^{*}\left(-\boldsymbol{u}_{n}\right)-\operatorname{tr}\left(\left(Z W+\mathbf{1} \boldsymbol{b}^{T}\right) U^{T}\right)
$$


Theorem 3. The optimal $\hat{\boldsymbol{u}}_{n}$ to the problem (12) is the negative mean vector of the sample $\boldsymbol{x}_{n}$ : $-\left.E_{\boldsymbol{\theta}_{n}}\left[\boldsymbol{x}_{n}\right]\right|_{\boldsymbol{\theta}_{n}=W^{T} \boldsymbol{z}_{n}+\boldsymbol{b}}$, which is also equal to $-\left.\frac{\partial A\left(\boldsymbol{\theta}_{n}\right)}{\partial \boldsymbol{\theta}_{n}}\right|_{\boldsymbol{\theta}_{n}=W^{T} \boldsymbol{z}_{n}+\boldsymbol{b}}$. Proof. To solve problem 12, we need the following result as proposed by [17]: for all canonical parameters $\gamma$ of the exponential family distribution of random variables $\boldsymbol{y} \in \mathcal{Y}, \sup _{\boldsymbol{\mu} \in \mathcal{M}}\left\{\langle\gamma, \boldsymbol{\mu}\rangle-A^{*}(\boldsymbol{\mu})\right\}$ is attained uniquely at the mean vector $\boldsymbol{\mu}^{*}$ specified by the moment matching condition:

$$
\boldsymbol{\mu}^{*}=\int_{\mathcal{Y}} \boldsymbol{y} p(\boldsymbol{y} \mid \gamma) d \boldsymbol{y}=E_{\boldsymbol{\gamma}}[Y]
$$

Similarly, consider an optimization problem: $\max _{\boldsymbol{v} \in \mathcal{M}^{\prime}}-\langle\boldsymbol{\gamma}, \boldsymbol{v}\rangle-A^{*}(-\boldsymbol{v})$, where $\mathcal{M}^{\prime}=\{m:-m \in \mathcal{M}\}$. Its maximum is attained at the vector $\boldsymbol{v}^{*}=$ $245-\boldsymbol{\mu}^{*}=-E_{\gamma}[Y]$.

According to this result, the optimal $\hat{\boldsymbol{u}}_{n}$ in 12 is obtained as the negative mean vector of the sample $\boldsymbol{x}_{n}:-\left.E_{\boldsymbol{\theta}_{n}}\left[\boldsymbol{x}_{n}\right]\right|_{\boldsymbol{\theta}_{n}=W^{T} \boldsymbol{z}_{n}+\boldsymbol{b}}$. Since the mean vector is further shown to be equal to the first derivative of the log-normalization factor $A(\cdot)$ according to Proposition 1 given in [17, we have $\hat{\boldsymbol{u}}_{n}=-\left.\frac{\partial A\left(\boldsymbol{\theta}_{n}\right)}{\partial \boldsymbol{\theta}_{n}}\right|_{\boldsymbol{\theta}_{n}=W^{T} \boldsymbol{z}_{n}+\boldsymbol{b}}$. One can also verify this solution by setting the first derivative of the objective function in 12 with respect to $\boldsymbol{u}_{n}$ equal to 0 .

For the outer minimization problem on $Z, W$, and $\boldsymbol{b}$, the objective function $f(Z, W, \boldsymbol{b}, \cdot)$ is quadratic as shown below:

$$
\begin{aligned}
& \left.f(Z, W, \boldsymbol{b}, .)\right|_{Z^{T} Z=I} \\
= & -\operatorname{tr}\left(\left(Z W+\mathbf{1 b}^{T}\right)(U+X)^{T}\right)+\lambda_{0}\left\|Z W+\mathbf{1} \boldsymbol{b}^{T}\right\|^{2}+\sum_{l=1}^{L} \lambda_{l}\left|W_{l}\right|+C_{0} \\
= & \lambda_{0}\left\|\frac{1}{2 \lambda_{0}}(X+U)-Z W-\mathbf{1} \boldsymbol{b}^{T}\right\|^{2}+\sum_{l=1}^{L} \lambda_{l}\left|W_{l}\right|+C_{1},
\end{aligned}
$$

where $C_{0}$ and $C_{1}$ are constant terms unrelated to $Z, W$, or $\boldsymbol{b}$. The minimization problem with respect to $Z, W$, and $\boldsymbol{b}$ has a similar form as the SPCA problem (7) with $P(\cdot)$ set as a $l_{1}$-norm penalty term and thus can be solved by the same strategy mentioned in Section 2.3 Although this problem involves non-convex constraints, an efficient solution can be achieved owing to the elegant problem structure. 
Specifically, in the $(t+1)$-th iteration, given an optimal $U^{t}, \boldsymbol{b}^{t+1}$ can be updated as:

$$
\boldsymbol{b}^{t+1}=\frac{1}{N}\left(\frac{1}{2 \lambda_{0}}\left(X+U^{t}\right)-Z^{t} W^{t}\right)^{T} \mathbf{1} .
$$

To update $Z$, the minimization problem with respect to $Z$ is:

$$
\begin{gathered}
\min _{Z: Z^{T} Z=I}\left\|\frac{1}{2 \lambda_{0}}(X+U)-\mathbf{1} \boldsymbol{b}^{T}-Z W\right\|^{2} \\
\equiv \min _{Z: Z^{T} Z=I}\left\|\frac{1}{2 \lambda_{0}}(X+U)^{T}-\boldsymbol{b} \mathbf{1}^{T}-W^{T} Z^{T}\right\|^{2} .
\end{gathered}
$$

Denote $Q$ as $\frac{1}{2 \lambda_{0}}(X+U)-\mathbf{1 b}^{T}$. We first compute the SVD of $Q^{t} W^{t T}=R \Lambda V^{T}$ and then update $Z^{t+1}$ by $R[, 1: L] V^{T}$ according to Theorem 1 .

To update $W$, the minimization problem with respect to $W$ is a LASSO problem:

$$
\begin{aligned}
& \min _{W}\left\|\frac{1}{2 \lambda_{0}}(X+U)-Z W-\mathbf{1 b}^{T}\right\|^{2}+\sum_{l=1}^{L} \frac{\lambda_{l}}{\lambda_{0}}\left|W_{l}\right| \\
\equiv & \min _{W}\|Q-Z W\|^{2}+\sum_{l=1}^{L} \frac{\lambda_{l}}{\lambda_{0}}\left|W_{l}\right| .
\end{aligned}
$$

The optimal $W_{l}^{t+1}$ for $l=1, \ldots, L$ is given by $\left(\left|Q^{t T} Z_{l}^{t}\right|-\frac{\lambda_{l}}{2 \lambda_{0}}\right)_{+} \operatorname{Sign}\left(Q^{t T} Z_{l}^{t}\right)$, where $Z_{l}$ denotes the $l$-th column of $Z$ corresponding to the $l$-th PC.

In summary, the detailed procedure for solving SePCA is summarized by

\section{Algorithm 1.}

\subsection{Computational complexity}

In the initialization step, it takes $O\left(N D^{2}\right)$ computational operations to compute the SVD. Our algorithm contains two main steps: maximization of $U$ and minimization of $Z, W$, and $\boldsymbol{b}$. Computing $U$ has the computational complexity of $O(N D L)$ in each iteration. In each iteration of optimizing $Z$, computing $Q W^{T}$ and the corresponding SVD has the complexity $O(N D L)$ and $O\left(N L^{2}\right)$, respectively. The estimation of $W$ using the soft-thresholding operation has the complexity of $O(N D L)$ in each iteration. In total, the computational complexity is $O\left(N D^{2}\right)+r O(N D L)$ if it takes $r$ iterations to converge. If $N<<D$, 


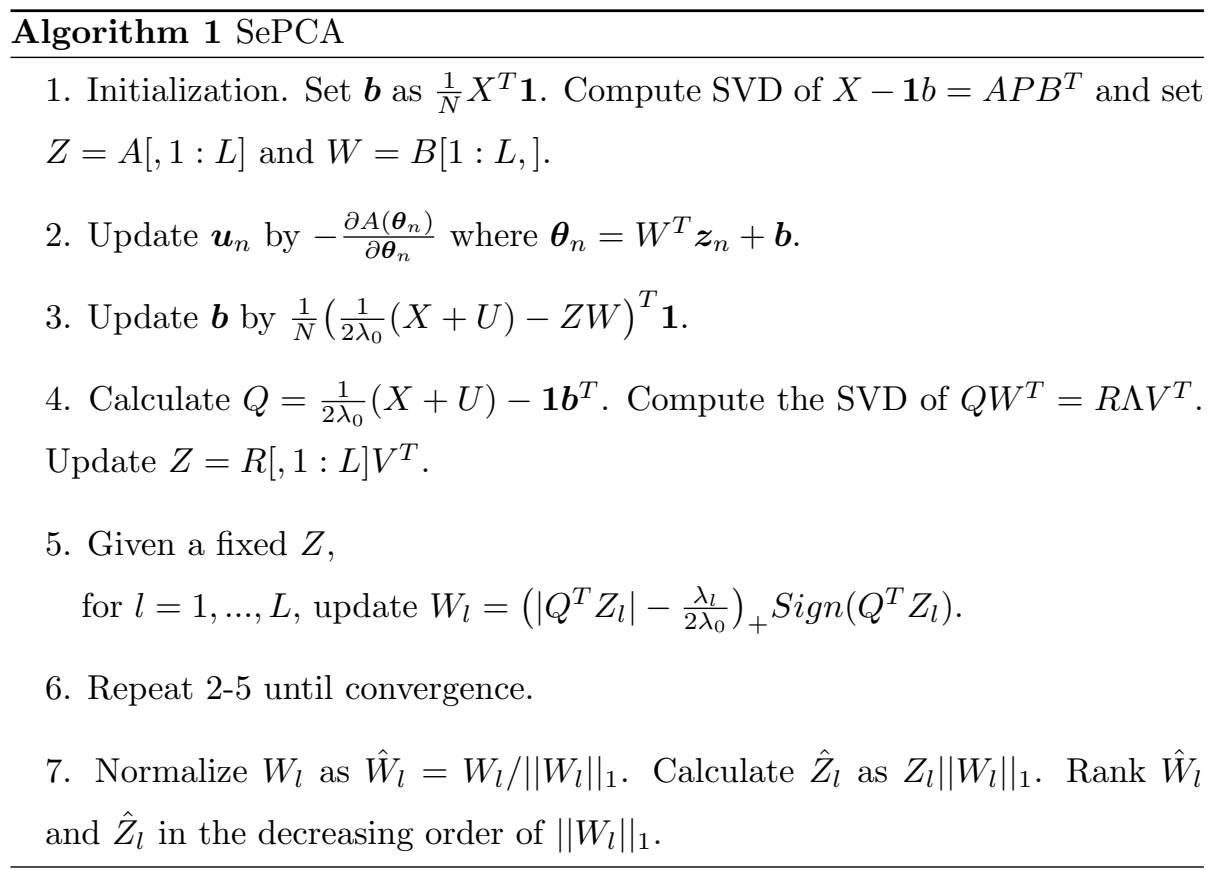

the cost of SVD in the initialization step can be reduced to $N^{2} D$ and the total computational complexity is $O\left(N^{2} D\right)+r O(N D L)$. The algorithm usually takes only a few iterations to converge according to our experience.

\subsection{Connections with $e P C A$ and $S P C A$}

Our SePCA is a generalization of both ePCA and SPCA and could reduce to them in special cases.

When $\lambda_{l}$ is set to 0 and the bias term $\boldsymbol{b}$ is dropped, SePCA reduces to an optimization problem with the same objective function and constraints as the ePCA problem presented in $[3$ but with a different alternating order of optimization with respect to $Z, W$, and $U$. Fortunately, these two problems are shown to be equivalent irrespective of the optimization order in [3]. Without the $l_{1}$-norm penalty term on $W$, our algorithm updates $W$ and $Z$ by $\frac{1}{2 \lambda_{0}} Z^{T}(X+U)$ and the first $L$ left vectors of matrix $X+U$ respectively, which conforms to the updates given by [3]. As for the $U$ update step, we directly update $U$ by a closed-form solution instead of the gradient ascent method used in [3]. 
Eventually, the gradient ascent approach will find the same solution since the objective function is concave with respect to $U$; however it will take longer time than our algorithm.

In the cases that the data set $X$ is assumed to be sampled from a Gaussian distribution, we have $A\left(\boldsymbol{\theta}_{n}\right)=\boldsymbol{\theta}_{n}^{T} \boldsymbol{\theta}_{n} / 2$ and $A^{*}\left(\boldsymbol{u}_{n}\right)=\boldsymbol{u}_{n}^{T} \boldsymbol{u}_{n} / 2$ correspondingly. 300 Consequently, $U$ is estimated as $-Z W$ when the data is centered. After substituting the estimated $U$ into the objective function in (11), we will arrive at the SPCA problem (7) with an elastic net penalty term.

\subsection{Choice of tuning parameters}

In Algorithm 1, the parameter $\lambda_{0}$ acts as a scaling factor for $W$, analogous to the role of tuning parameters for the $l_{2}$-norm term in the elastic net in Zou et al's SPCA. The default choice of $\lambda_{0}$ can be 1 . For simplicity, we treat $\lambda_{l}$ for different principal components equally and then we only need to determine one common parameter $\lambda$. The $l_{1}$-norm penalty parameter controls the model complexity. To compromise the goodness of fit and model complexity, we use the Bayesian Information Criterion (BIC) to achieve the maximum likelihood with the most model generalization. $\lambda$ is chosen by minimizing the following BIC criterion:

$$
B I C=-2 \log \hat{\ell}(U, Z, W, \boldsymbol{b})+\log (N D) \times m(\lambda),
$$

where $\hat{\ell}$ is the estimated log-likelihood and $m(\lambda)$ is the number of free parameters to be estimated: $m(\lambda)=N D+N K+D+|W(\lambda)|$ where $N D$ is the total number of elements of $U, N K$ is the total number of elements of $Z, D$ is the length of the vector $\boldsymbol{b}$, and $|W(\lambda)|$ is the number of non-zero loadings in $W$ when the penalty parameter is $\lambda$.

\section{Experimental results}

We have investigated the performance of our SePCA model and the efficiency of the algorithm via simulated studies and real-world applications. The 
simulation studies aim to examine the accuracy and computational efficiency of SePCA on binary data and count data. These performances are further investigated by real-world applications in image clustering and population stratification that involve count data and binary data respectively. We show the superiority of SePCA in analyzing non-Gaussian data by comparing it with Zou et al.'s SPCA [5]. For binary data cases, we also compare SePCA with a previous sparse logistic PCA method solved by coordinate descent Majorization-Minimization algorithm [18, which is denoted as SLPCA_MM in this paper. We denote SePCA under a certain exponential family distribution such as Bernoulli distribution or Poisson distribution as SePCA_Bern or SePCA_Pois, respectively. In addition, we also compare our SePCA with ePCA to investigate its advantage in improving the results by regularization of the loading vectors when $N<D$. The advantage of our closed-form updating rules in computational efficiency also has been examined by comparing ePCA with our SePCA without sparsity regularization for fair comparison.

\subsection{Simulation studies}

\subsubsection{Simulation design}

In this set of simulation experiments, we have studied the performance of SePCA in dimension reduction for binary data and count data respectively. For each data type, we have simulated a matrix $X$ of $N$ independent $D$-dimensional samples, where each element $X_{n d}$ is simulated using its probability distribution function of the canonical parameter $\Theta_{n d}$. As we introduced, $\Theta$ is parameterized as $Z W+\mathbf{1} \boldsymbol{b}^{T}$, where $Z$ is a $N \times L$ principal component score matrix and $W$ is a $L \times D$ principal component loading matrix. $L$ is the number of principal components, which is set to 2 in the simulation studies. For simplicity, we set the bias vector $\boldsymbol{b}$ to 0 . The loading matrix $W$ is generated as a sparse matrix, in which only $W[1,1: 20]$ and $W[2,21: 40]$ are set to 1 while the other elements are all 0 . Each loading vector $W_{l}$ is further normalized with a unit $l_{2}$-norm. The principal component scores $Z_{l}$ are generated randomly from a Gaussian distribution with zero mean and a variance of $\sigma_{l}^{2}$. 
To have a thorough evaluation, we have studied multiple simulated data sets with various $N, D$, and $\sigma_{l}^{2}$. We considered 8 different settings of $(N, D)$ where $N$ has two choices: 100, 200; and $D$ has four choices: 50, 100, 200, 500, respectively. Since the variance $\sigma_{l}^{2}$ measures the signal level of the $l$-th $\mathrm{PC}$, we 355 set the PC variance relative to a suitably defined baseline noise level by $\sigma_{l}^{2}=$ $S N R_{l} \times \sigma_{0}^{2}$ (baseline noise level), where $S N R_{l}$ is the signal-to-noise ratio for the $l$-th PC. The baseline noise level is defined as the variance of PC scores from binary data under the unstructured model 9 . The details about calculating the baseline noise level can be found in Section 6.1 of [9]. We use $S N R=\left(S N R_{1}\right.$, $\left.S N R_{2}\right)$ to set the variances for the two PCs. Finally, for each data type, we have simulated 100 data sets for each of these 8 settings under $S N R=(3,2)$ and $(5,3)$ respectively.

The performances of our SePCA_Bern and SePCA_Pois are evaluated based on three criteria: 1) the maximum angle between the estimated PC loadings and true PC loadings, 2) true positive selection rate - the percentage of the true 40 non-zero (informative) elements in the simulated ground-truth loading matrix that are estimated as non-zeros, and 3) false positive selection rate the percentage of the true zero (non-informative) elements in the ground-truth loading matrix that are estimated as non-zeros. The angle measures the reconstruction accuracy for PC loading vectors in the perspective of their spanning direction, while the other two measure the accuracy in the perspective of sparse structure for correct interpretation of contributing variables in PCs. A good method is expected to achieve small angle and false positive selection rate when the true positive selection rate arrives at $100 \%$.

\subsubsection{Binary data study}

In this study, each element $X_{n d}$ is sampled from a Bernoulli distribution with the success probability: $\frac{\exp \left(\Theta_{n d}\right)}{1+\exp \left(\Theta_{n d}\right)}$.

By specifying $A(\theta)$ as $\log (1+\exp (\theta))$, we have applied SePCA_Bern to the simulated data sets and have compared its performance with SPCA, SLPCA_MM and ePCA [19]. The smallest angles for different data sets shown in Tables 1 
and 2 suggest that our SePCA_Bern outperforms the others for all cases in reconstructing more accurate PC loadings. A visualization of the angles for these methods across all 100 replicates under the first setting with $N=100, D=50$, and $S N R=(3,2)$ is illustrated in Figure 2 . One can observe that SePCA_Bern always achieves the smallest angles among these methods, which implies the consistency of our superiority. By observing true positive selection rates and false positive selection rates for the first three methods, we see that almost all of them have selected all the true non-zero elements to predict the PC scores while SLPCA_MM and SPCA selected more additional zero (non-informative) elements, which indeed have no contribution to the true PCs. This suggests that the sparse structure of our estimated PC loadings by SePCA_Bern is the closest to the ground truth. The superiority of SePCA_Bern over SPCA and SLPCA_MM is believed to benefit from the explicit modeling of binary data and from the direct optimization of the objective function through conjugate dual, respectively. Compared with ePCA, our method significantly improves the results by regularizing the loading vectors for these data sets with the latent sparse structures.

All the experiments in this article were run on a Mac OS X with a $2.5 \mathrm{GHz}$ Intel Core i5 processor. When investigating computational efficiency, SePCA_Bern takes much less running time than SPCA especially for higher dimensional data since SPCA requires the expensive computation of Gram matrix $X^{T} X$, which is avoided in SePCA_Bern. Our SePCA_Bern also has superiority over ePCA in computational efficiency due to the closed-form updating rules. We further compare ePCA and SePCA_Bern without adding the sparsity penalty term in the running time with regard to different data dimensions in order to thoroughly investigate how significant advantages the closed-form updating rules make. The results are shown in Figure 3 for the case with $N=100$ and $S N R=(3,2)$, in which we have observed significant difference between ePCA and our SePCA_Bern: ePCA takes about 30 times longer than our method when 410 $D=100,000$. 
Table 1: Comparison of the performance of SPCA, SLPCA_MM, SePCA_Bern and ePCA on simulated binary data at $S N R=(3,2)$. The average (standard deviation) of the running time, maximum angle of PC loadings, true positive selection rate and false positive selection rate over 100 simulations are presented for these methods.

\begin{tabular}{|c|c|c|c|c|c|c|}
\hline $\mathrm{N}$ & $\mathrm{D}$ & Method & Time(sec.) & Angle(degree) & True Pos.(\%) & False Pos. $(\%)$ \\
\hline \multicolumn{7}{|c|}{$\mathrm{SNR}=(3,2)$} \\
\hline \multirow[t]{16}{*}{100} & 50 & SPCA & $0.70(0.60)$ & $11.70(4.78)$ & $100(0)$ & $4.83(2.48)$ \\
\hline & & SLPCA_MM & $0.48(0.34)$ & $7.88(1.85)$ & $100(0)$ & $6.63(3.48)$ \\
\hline & & SePCA_Bern & $0.09(0.02)$ & $5.28(0.99)$ & $100(0)$ & $1.00(1.14)$ \\
\hline & & $\mathrm{ePCA}$ & $0.90(0.14)$ & $17.4(2.28)$ & $100(0)$ & $16.7(0)$ \\
\hline & 100 & SPCA & $1.37(0.92)$ & $17.1(5.55)$ & $100(0)$ & $11.7(2.62)$ \\
\hline & & SLPCA_MM & $0.672(0.26)$ & $8.51(1.37)$ & $100(0)$ & $10.2(2.77)$ \\
\hline & & SePCA_Bern & $0.17(0.03)$ & $6.96(1.69)$ & $100(0)$ & $0.36(0.49)$ \\
\hline & & $\mathrm{ePCA}$ & $1.83(0.29)$ & $17.5(1.86)$ & $100(0)$ & $37.5(0)$ \\
\hline & 200 & SPCA & $3.08(3.05)$ & $25.6(6.03)$ & $99.9(0.49)$ & $12.1(2)$ \\
\hline & & SLPCA_MM & $0.83(0.63)$ & $6.84(1.08)$ & $100(0)$ & $0.74(0.28)$ \\
\hline & & SePCA_Bern & $0.4(0.14)$ & $6.07(1.14)$ & $100(0)$ & $0.08(0.09)$ \\
\hline & & ePCA & $2.44(0.48)$ & $17.9(1.77)$ & $100(0)$ & $44.4(0)$ \\
\hline & 500 & SPCA & $9.05(7.33)$ & $39.5(5.41)$ & $96.8(3.11)$ & $8.48(0.95)$ \\
\hline & & SLPCA_MM & $1.61(0.68)$ & $7.82(1.33)$ & $100(0)$ & $0.69(0.45)$ \\
\hline & & SePCA_Bern & $0.98(0.11)$ & $7.26(1.42)$ & $100(0)$ & $0.11(0.21)$ \\
\hline & & $\mathrm{ePCA}$ & $4.46(1.69)$ & $19.2(1.76)$ & $100(0)$ & $47.9(0)$ \\
\hline \multirow[t]{16}{*}{200} & 50 & SPCA & $0.75(0.84)$ & $5.93(1.59)$ & $100(0)$ & $8.33(2.94)$ \\
\hline & & SLPCA_MM & $0.9(0.60)$ & $6.09(1.21)$ & $100(0)$ & $7.1(4.06)$ \\
\hline & & SePCA_Bern & $0.3(0.03)$ & $3.31(0.49)$ & $100(0)$ & $0.97(1.14)$ \\
\hline & & $\mathrm{ePCA}$ & $0.87(0.13)$ & $12(1.29)$ & $100(0)$ & $16.7(0)$ \\
\hline & 100 & SPCA & $5.32(7.98)$ & $10.1(1.71)$ & $100(0)$ & $21.6(2.17)$ \\
\hline & & SLPCA_MM & $3.79(4.03)$ & $7.38(0.94)$ & $100(0)$ & $7.55(3.8)$ \\
\hline & & SePCA_Bern & $1.03(1.18)$ & $5.53(1.05)$ & $100(0)$ & $0.36(0.55)$ \\
\hline & & $\mathrm{ePCA}$ & $1.83(0.87)$ & $13.3(1.27)$ & $100(0)$ & $37.5(0)$ \\
\hline & 200 & SPCA & $2.23(1.18)$ & $15.6(1.66)$ & $100(0)$ & $10.9(1.45)$ \\
\hline & & SLPCA_MM & $1.28(0.66)$ & $8.26(1.22)$ & $100(0)$ & $0.77(0.27)$ \\
\hline & & SePCA_Bern & $1.3(0.89)$ & $7.47(1.28)$ & $100(0)$ & $0.09(0.10)$ \\
\hline & & $\mathrm{ePCA}$ & $1.81(0.97)$ & $20.8(1.8)$ & $100(0)$ & $44.4(0)$ \\
\hline & 500 & SPCA & $9.28(4.18)$ & $20.6(2.08)$ & $100(0)$ & $9.16(1.04)$ \\
\hline & & SPCA_MM & $2.45(0.07)$ & $10.9(1.78)$ & $100(0)$ & $0.731(0.4)$ \\
\hline & & SLPCA_Bern & $3.16(0.08)$ & $10.6(1.97)$ & $100(0)$ & $0.11(0.19)$ \\
\hline & & ePCA & $4.97(4.45)$ & $23.5(1.84)$ & $100(0)$ & $47.9(0)$ \\
\hline
\end{tabular}


Table 2: Comparison of the performance of SPCA, SLPCA_MM, SePCA_Bern and ePCA on simulated binary data at $S N R=(5,3)$. The average (standard deviation) of the running time, maximum angle of PC loadings, true positive selection rate and false positive selection rate over 100 simulations are presented for these methods.

\begin{tabular}{|c|c|c|c|c|c|c|}
\hline $\mathrm{N}$ & $\mathrm{D}$ & Method & Time(sec.) & Angle(degree) & True Pos.(\%) & False Pos.(\%) \\
\hline \multicolumn{7}{|c|}{$\mathrm{SNR}=(5,3)$} \\
\hline \multirow[t]{16}{*}{100} & 50 & SPCA & $0.67(0.60)$ & $11.9(4.96)$ & $100(0)$ & $4.1(2.54)$ \\
\hline & & SLPCA_MM & $0.38(0.13)$ & $7.38(2)$ & $100(0)$ & $7.28(3.82)$ \\
\hline & & SePCA_Bern & $0.09(0.01)$ & $4.36(0.93)$ & $100(0)$ & $0.98(1.19)$ \\
\hline & & $\mathrm{ePCA}$ & $0.97(0.11)$ & $17.1(2.02)$ & $100(0)$ & $16.7(0)$ \\
\hline & 100 & SPCA & $1.17(0.87)$ & $17.8(5.58)$ & $100(0)$ & $10.3(2.67)$ \\
\hline & & SLPCA_MM & $0.69(0.24)$ & $7.18(1.11)$ & $100(0)$ & $8.99(3.24)$ \\
\hline & & SePCA_Bern & $0.17(0.03)$ & $5.38(1.18)$ & $100(0)$ & $0.37(0.51)$ \\
\hline & & $\mathrm{ePCA}$ & $1.99(0.25)$ & $16.2(1.74)$ & $100(0)$ & $37.5(0)$ \\
\hline & 200 & SPCA & $2.81(2.34)$ & $26.3(5.8)$ & $99.9(0.55)$ & $11.4(2.04)$ \\
\hline & & SLPCA_MM & $0.81(0.31)$ & $6.52(1.3)$ & $100(0)$ & $0.75(0.69)$ \\
\hline & & SePCA_Bern & $0.41(0.05)$ & $5.69(1.07)$ & $100(0)$ & $0.1(0.18)$ \\
\hline & & $\mathrm{ePCA}$ & $2.67(0.34)$ & $16.3(1.59)$ & $100(0)$ & $44.4(0)$ \\
\hline & 500 & SPCA & $7.64(4.29)$ & $40.6(5.16)$ & $95.3(3.67)$ & $8.13(0.91)$ \\
\hline & & SLPCA_MM & $1.54(0.1)$ & $5.75(1)$ & $100(0)$ & $0.75(0.30)$ \\
\hline & & SePCA_Bern & $0.97(0.05)$ & $4.91(0.98)$ & $100(0)$ & $0.08(0.09)$ \\
\hline & & $\mathrm{ePCA}$ & $4.56(2.88)$ & $17.7(1.61)$ & $100(0)$ & $47.9(0)$ \\
\hline \multirow[t]{16}{*}{200} & 50 & SPCA & $1.4(2.81)$ & $5.52(1.46)$ & $100(0)$ & $7.98(2.66)$ \\
\hline & & SLPCA_MM & $1.26(1.45)$ & $5.54(1.27)$ & $100(0)$ & $8.07(4.54)$ \\
\hline & & SePCA_Bern & $0.57(0.69)$ & $2.68(0.46)$ & $100(0)$ & $1.02(1.16)$ \\
\hline & & $\mathrm{ePCA}$ & $1.08(0.22)$ & $11.6(1.34)$ & $100(0)$ & $16.7(0)$ \\
\hline & 100 & SPCA & $5.17(7.27)$ & $9.15(1.75)$ & $100(0)$ & $20.2(2.1)$ \\
\hline & & SLPCA_MM & $5.54(5.01)$ & $6.2(0.81)$ & $100(0)$ & $7.5(3.57)$ \\
\hline & & SePCA_Bern & $1.71(1.77)$ & $4.28(0.68)$ & $100(0)$ & $0.4(0.55)$ \\
\hline & & $\mathrm{ePCA}$ & $3.00(2.18)$ & $12.3(1.21)$ & $100(0)$ & $37.5(0)$ \\
\hline & 200 & SPCA & $9.59(11.1)$ & $15.9(1.67)$ & $100(0)$ & $24.5(1.75)$ \\
\hline & & SLPCA_MM & $1.61(1.51)$ & $8.3(1.31)$ & $100(0)$ & $0.74(0.44)$ \\
\hline & & SePCA_Bern & $1.24(0.80)$ & $7.46(1.39)$ & $100(0)$ & $0.09(0.16)$ \\
\hline & & $\mathrm{ePCA}$ & $4.02(4.47)$ & $17.3(1.53)$ & $100(0)$ & $44.4(0)$ \\
\hline & 500 & SPCA & $7.03(4.29)$ & $19(2.33)$ & $100(0)$ & $8.14(0.98)$ \\
\hline & & SLPCA_MM & $4.63(4.62)$ & $6.69(0.88)$ & $100(0)$ & $0.76(0.26)$ \\
\hline & & SePCA_Bern & $4.36(3.69)$ & $5.62(0.86)$ & $100(0)$ & $0.09(0.09)$ \\
\hline & & $\mathrm{ePCA}$ & $6.12(2.49)$ & $19.8(1.37)$ & $100(0)$ & $47.9(0)$ \\
\hline
\end{tabular}




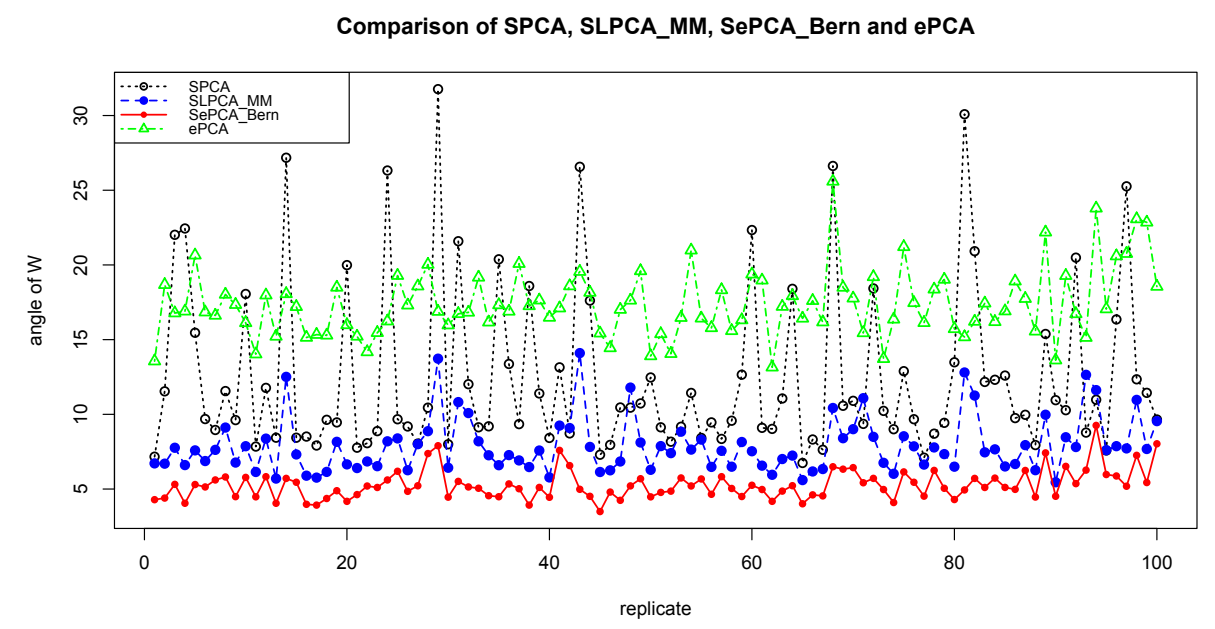

Figure 2: Plot of the maximum angle of PC loadings for SPCA, SLPCA_MM, SePCA_Bern and ePCA across 100 replicates at $N=100, D=50$, and $S N R=(3,2)$.

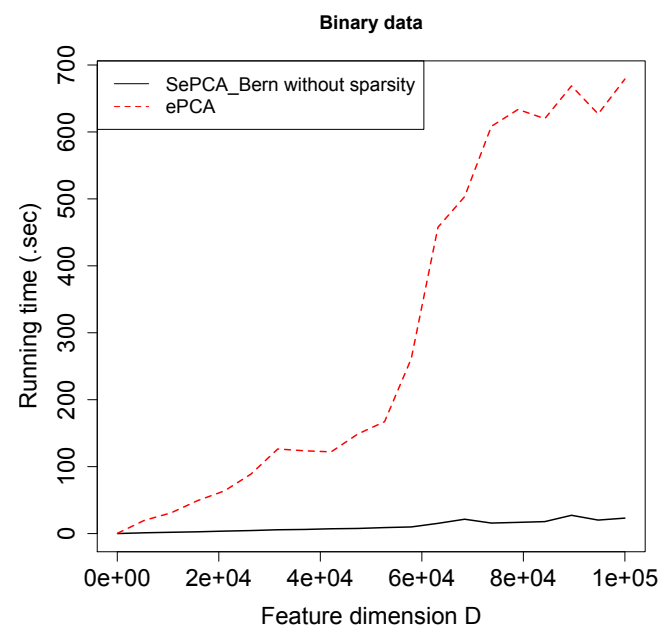

Figure 3: Plot of the running time versus feature dimension for ePCA and SePCA_Bern without sparsity regularization at $N=100$ and $S N R=(3,2)$.

\subsubsection{Count data study}

In this study, each element $X_{n d}$ is sampled from a Poisson distribution with the mean $\exp \left(\Theta_{n d}\right)$. We have implemented SePCA_Pois on these count data sets 
Table 3: Comparison of the performance of SPCA, SLPCA_MM, SePCA_Pois and ePCA on simulated count data at $N=100, D=50$, and $S N R=(3,2)$. The average (standard deviation) of the running time, maximum angle of $\mathrm{PC}$, true positive selection rate and false positive selection rate over 100 simulations are presented for these methods.

\begin{tabular}{|c|c|c|c|c|c|c|}
\hline $\mathrm{N}$ & D & Method & Time(sec.) & Angle(degree) & True Pos.(\%) & False Pos.(\%) \\
\hline \multicolumn{7}{|c|}{$\mathrm{SNR}=(3,2)$} \\
\hline \multirow[t]{4}{*}{100} & 50 & SPCA & $0.065(0.013)$ & $1.47(1.58)$ & $100(0)$ & $0(0)$ \\
\hline & & SLPCA_MM & $0.092(0.273)$ & $74.5(32.3)$ & $58.9(18.7)$ & $0(0)$ \\
\hline & & SePCA_Pois & $0.061(0.006)$ & $1.17(0.65)$ & $100(0)$ & $0(0)$ \\
\hline & & $\mathrm{ePCA}$ & $5.71(1.61)$ & $3.19(0.908)$ & $100(0)$ & $16.7(0)$ \\
\hline
\end{tabular}

with $A(\theta)$ set to $\exp (\theta)$. We also have compared it with SPCA, SLPCA_MM and ePCA [19] based on the criteria of running time, maximum angle of PC loadings, true positive selection rate, and false positive selection rate. In order to apply SLPCA_MM on a count data set, we set a threshold at the half of its maximum value to dichotomize each element. We show the results of these methods for the 100 replicate simulated data sets with $N=100, D=50$, and $S N R=(3,2)$ in Table 3 As shown in this table, SePCA_Pois again achieves the smallest angle and false positive selection rate, which suggests that our method obtains the most accurate PC loadings with the sparse structure closest to the ground truth. SLPCA_MM have had pretty bad performance due to the information loss from dichotomization. Figure 4 has further verified the advantage of closed-form updating rules of our method compared with ePCA on count data. We can observe the significant speedup of our SePCA_Pois. In this set of experiments, ePCA require almost three magnitudes longer running time than our method when $D=1,000$.

In summary, both the sets of simulation results suggest that our SePCA outperforms the others with an appealing model that considers the appropriate assumption of data distribution and regularization of loading vectors simultaneously. The optimization strategy also leads to better results than the ones by the MM algorithm. With the efficient closed-form updating rules, our SePCA algorithm significantly improves the computational efficiency compared with all the other methods. 


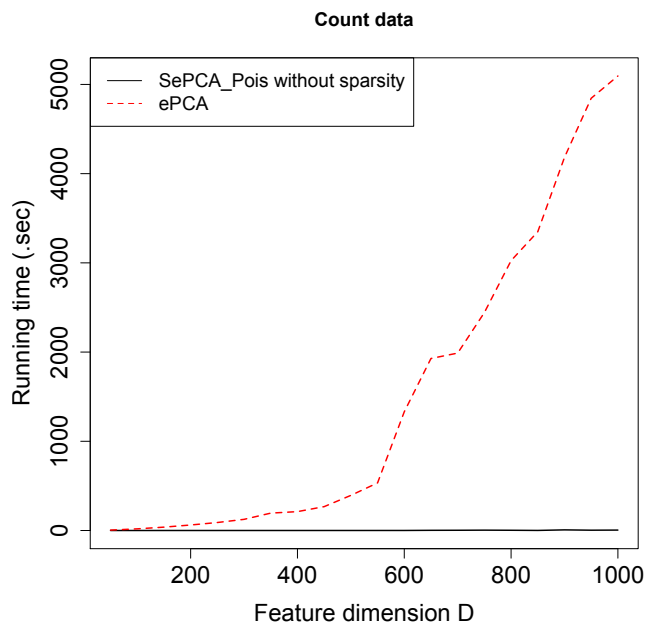

Figure 4: Plot of the running time versus feature dimension for ePCA and SePCA_Pois without sparsity regularization at $N=100$ and $S N R=(3,2)$.

\subsection{Face image clustering}

As image data is usually high-dimensional and involves redundant information caused by locally related pixels, it is desirable to reduce the dimension and redundancy via penalized latent factor analysis with variable selection to pursue better performance and interpretation. We have applied SePCA on a data set from the Yale image database 20] and have performed k-means clustering on the obtained PCs to compare the clustering performance for our method and other PCA methods.

There are 11 different images for each of 15 distinct subjects in the Yale database. The images of each subject vary with different facial expressions or configurations: center-light, w/glasses, happy, left-light, w/no glasses, normal, right-light, sad, sleepy, surprised, and wink. We have randomly selected a data set containing 44 images from 4 subjects: 1, 4, 6 and 8, corresponding to 4 ground-truth clusters respectively. We have used a center region of $128 \times 128$ (= $16,384)$ pixels from the original images by removing the redundant white background pixels. These images are shown in Figure 5 . This data set can be 
represented by a $44 \times 16,384$ matrix with each row corresponding to one image. Our goal is to cluster these images into 4 clusters corresponding to the four selected subjects. Due to the high dimensionality of of these face images, we aim to perform the clustering in a lower $L$-dimensional subspace constructed by the generalized principal components obtained by performing SePCA_Pois on the images, where the pixel intensities are considered as count data following Poisson distributions. The irrelevant or redundant pixels are taken care by sparsity regularization of the PC loading vectors.

We have studied the clustering performance at $L=1,2,3$, respectively. The clustering accuracy is calculated as the proportion of correctly assigned labels based on the best match with true labels [21, 22. We have also applied SPCA on this data set for comparison of the clustering performance. In this experiment, ePCA is not included because it fails to perform on this high dimensional data set due to required high computation resources, while our method can finish the task in half an hour with our closed-form updating rules. In addition to the clustering performance, we also have checked the number of selected pixels by calculating the sparsity rate of the loading vectors. The sparsity rate is reflected by the "Total(\%)" column in Table 4 , which calculates the number of non-zero 470 elements in all the PC loadings and its percentage. The number of non-zeros in each PC loading as well as its percentage are shown in the following columns respectively. The results in Table 4 have shown that SePCA_Pois has achieved higher or competitive clustering accuracy than SPCA at a larger sparsity rate in all the three cases. These observations suggest that the appropriate assumption of the data distribution gives rise to more appealing dimension reduction results using fewer variables than standard SPCA. The visualization of 2-PC projections for SePCA_Pois and SPCA are shown in Figure6, which demonstrates that the projections obtained from SePCA_Pois have more obvious clustering boundary and smaller within-cluster distance. 


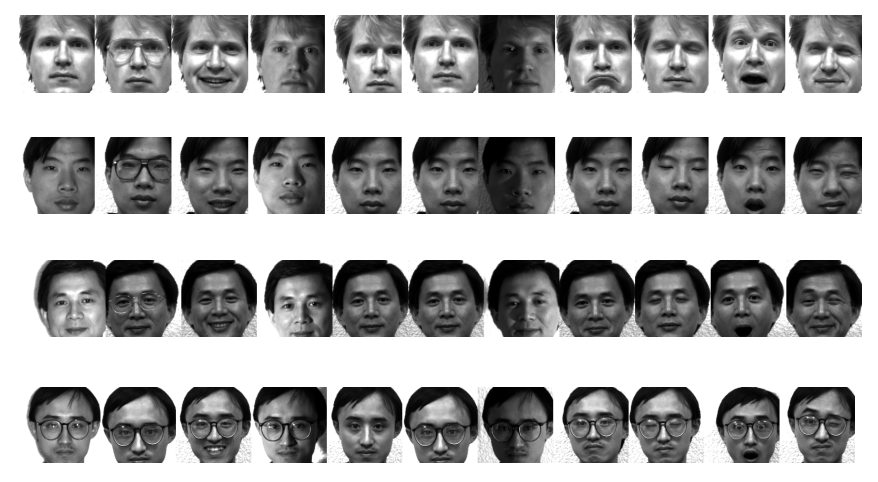

Figure 5: Visualization of the 44 Yale images from subjects 1, 4, 6 and 8 shown by four rows correspondingly.

Table 4: Comparison of the clustering performance by SPCA and SePCA_Pois on Yale data. The clustering accuracy and the number of non-zero variables (percentage) in all the loading vectors as well as these indices in each loading vector are presented for both methods.

\begin{tabular}{lcllll}
\hline Method & Acc. & Total $(\%)$ & PC1 $(\%)$ & PC2(\%) & PC3(\%) \\
\hline L=1 & & & & & \\
SPCA & 0.61 & $7064(43.1)$ & $7064(43.1)$ & - & - \\
SePCA_Pois & 0.61 & $6023(36.8)$ & $6023(36.8)$ & - & - \\
\hline L=2 & & & & & \\
SPCA & 0.64 & $7693(47.0)$ & $7076(43.2)$ & $4916(30.0)$ & - \\
SePCA_Pois & 0.70 & $6998(42.7)$ & $5666(34.6)$ & $5320(32.5)$ & - \\
\hline L=3 & & & & & \\
SPCA & 0.84 & $7906(48.3)$ & $6286(38.3)$ & $5994(36.6)$ & $4924(30.1)$ \\
SePCA_Pois & 0.84 & $7640(46.6)$ & $6133(37.4)$ & $5414(33.0)$ & $3762(23.0)$ \\
\hline
\end{tabular}



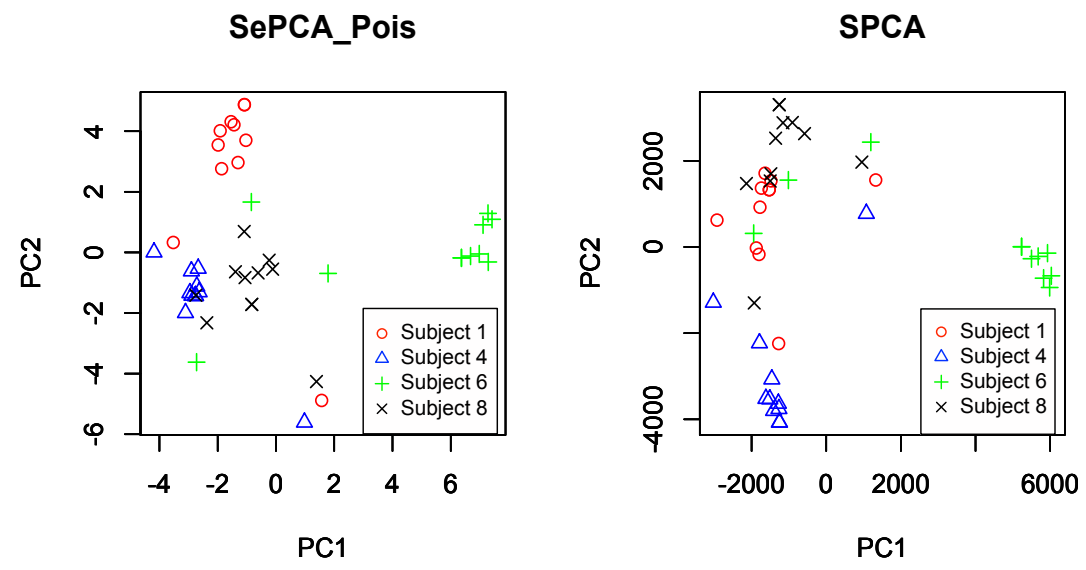

Figure 6: Visualization of the distribution of the 44 Yale images from 4 subjects in 2-PC subspace.

We have applied our algorithm on Single Nucleotide Polymorphism (SNP) data from the International HapMap Project (HapMap3) 23] to analyze the subpopulation structure. This dataset contains 1,301 samples from 11 populations of European ancestry, Asian ancestry, and African ancestry. Those samples from the same population tend to have a common pattern of genetic variation expressed by SNPs, which can be detected by clustering. We treat SNP data as binary data with 0 representing the most prevalent homogeneous base pair (wild-type) and 1 representing the other genotypes (mutant with minor alleles). Considering millions of SNPs are genotyped in the data set, we reduce the number of SNPs by quality control via removing SNPs and samples with the minor allele frequency less than 0.05, excluding regions with strong linkage disequilibrium (LD) such as Major histocompatibility complex (MHC), and performing LD pruning to make sure pairs of variants in a window size of $50 \mathrm{~kb}$ have correlation value $r^{2}$ less than 0.2. After randomly sampling 5,000

SNPs and removing the SNPs with missing values, we have a smaller data set 
with 1,184 samples and 748 SNPs for clustering analysis. Since these 11 populations can be clearly grouped into three clusters corresponding to the three categories of ancestry, we only present our results on identifying the four subpopulations with the African ancestry: African ancestry in Southwest USA (ASW); Luhya in Webuye, Kenya (LWK); Maasai in Kinyawa, Kenya (MKK) and Yoruba in Ibadan, Nigeria (YRI) with 83, 90, 171, and 166 samples, respectively. Thus, we have 511 samples in this study in total. We have applied SPCA, SLPCA_MM, SePCA_Bern, and ePCA for clustering and found that SLPCA_MM and SePCA_Bern have competitive clustering accuracy and sparsity in all three cases demonstrated by Table 5 . It suggests that SePCA_Bern has no obvious improvement compared with SLPCA_MM when a fairly large portion of variables contribute to the projections. However, both of them have considerable improvement over SPCA in clustering accuracy at the competitive sparsity rate owing to their explicit modelings of binary data. We also find that our method improves the clustering accuracy compared with ePCA by regularizing the loading vectors to assign more appropriate weights for informative SNPs.

To examine the computational efficiency of SLPCA_MM, SePCA_Bern, and ePCA, we have recorded their running time for a different number of PCs. As shown in Figure 7, the running time of ePCA is one magnitude longer than that of the other methods, and will increase dramatically with the increasing number of PCs. Our SePCA has the running time quite insensitive to the number of PCs and outperforms SLPCA_MM with the increasing gain in computational efficiency as the number of PCs increases. The increasing trend in the running time of SLPCA_MM is believed as a result of the one-by-one coordinate-descent optimization for PCs. Although one may notice that the coordinate-descent update in SLPCA_MM will cost slightly less than SVD in our algorithm when quite a few PCs are computed, we can further speed up our algorithm by doing a truncated SVD when the required number of PCs is lower than the full rank 525 of the data matrix. 
Table 5: Comparison of the clustering performance by SPCA, SLPCA_MM, SePCA_Bern, and ePCA on HapMap data. The clustering accuracy and the number of non-zero variables (percentage) in all the loading vectors as well as these indices in each loading vector are presented for these methods.

\begin{tabular}{llllll}
\hline Methods & Acc. & Total $(\%)$ & PC1 $(\%)$ & PC2(\%) & PC3(\%) \\
\hline L=1 & & & & & \\
SPCA & 0.81 & $502(67.1)$ & $502(67.1)$ & - & - \\
SLPCA_MM & 0.85 & $567(75.8)$ & $567(75.8)$ & - & - \\
SePCA_Bern & 0.85 & $553(73.9)$ & $553(73.9)$ & - & - \\
ePCA & 0.79 & - & - & - & - \\
\hline L=2 & & & & & \\
SPCA & 0.81 & $633(84.6)$ & $496(66.3)$ & $494(66.0)$ & - \\
SLPCA_MM & 0.85 & $659(88.1)$ & $568(75.9)$ & $420(56.1)$ & - \\
SePCA_Bern & 0.85 & $650(86.9)$ & $553(73.9)$ & $402(53.7)$ & - \\
ePCA & 0.79 & - & - & - & - \\
\hline L=3 & & & & & \\
SPCA & 0.66 & $688(92.0)$ & $489(65.4)$ & $483(64.6)$ & $489(65.4)$ \\
SLPCA_MM & 0.68 & $708(94.6)$ & $568(75.9)$ & $440(58.8)$ & $420(56.1)$ \\
SePCA_Bern & 0.68 & $700(93.6)$ & $554(74.1)$ & $414(55.3)$ & $400(53.5)$ \\
ePCA & 0.66 & - & - & - & - \\
\hline
\end{tabular}

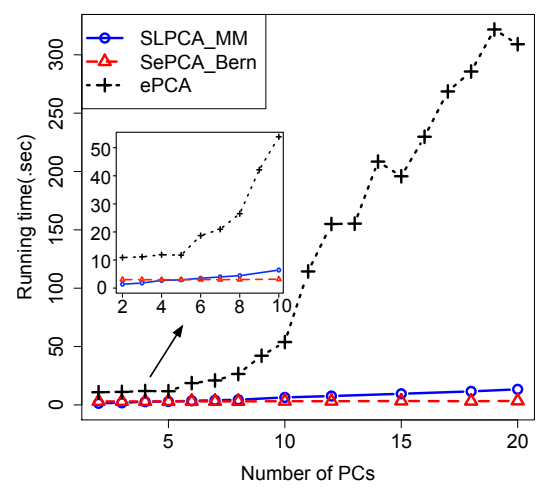

Figure 7: Comparison of the running time versus the number of PCs for SLPCA_MM, SePCA_Bern, and ePCA on HapMap data. 


\section{Conclusions}

We have proposed a new sparse model of exponential family PCA — SePCA to enable variable selection in low-dimensional analysis of exponential family data for better systematic interpretation in real-world applications. In compartial family data. Our experimental results have empirically demonstrated that SePCA can achieve more accurate and sparse PC loadings compared with Zou et al.'s SPCA via explicit modeling of those non-Gaussian data. By optimizing the exact log-likelihood function, SePCA_Bern outperforms an existing logistic loadings for high-dimensional binary data. Our method also improves the reconstruction accuracy of ePCA when the data dimension is larger than the sample number or the data has a latent sparse structure. Moreover, SePCA achieves much higher computational efficiency compared with SPCA, SLPCA_MM, and which leads to the efficient closed-form updating rules.

The proposed SePCA model is quite general to be applied on any type of high-dimensional data following the distributions from the exponential family. The choice of the form of exponential family distributions can be made by examining the data distribution before running any PCA methods. When the data follow the Gaussian distribution, our specific form of SePCA naturally reduces to the sparse PCA [6]. It is convenient to use the Bernoulli distribution for binary data and Poisson distribution for count data when applying the SePCA method. Our SePCA is generally preferred to ePCA when there is a large numinterpretation of results.

Our model is flexible and highly extensible. With integration of additional label information into the current framework, it can be extended to a supervised-learning model to solve classification or regression problems involving high-dimensional exponential family data. The dual-transformation strategy 
is still applicable for this problem due to the similar form of models shared by ePCA and GLMs. Closed-form updating rules are still available and efficient for these supervised learning problems. This model provides a way for hierarchical analysis based on several dominant variables. It can simultaneously estimate the principal component effects as well as the individual effects of the dominant variables in association analyses. Moreover, one can also apply other regularization terms on PC loadings to achieve smoothness or perform graph-regularized learning.

\section{Acknowledgements}

The project was supported in part by Award R21DK092845 from the National Institute of Diabetes and Digestive and Kidney Diseases (NIDDK), National Institutes of Health (NIH). XQ is also partially supported by NSF Awards \#1447235, \#1547557, and \#1553281.

\section{References}

[1] M. Collins, S. Dasgupta, R. E. Schapire, A generalization of principal component analysis to the exponential family, Advanced in Neural Information Processing System 14 (2002) 617-642.

[2] A. I. Schein, L. K. Saul, L. H. Ungar, A generalized linear model for principal component analysis of binary data, In Proceedings of the 9 th International Workshop on Artificial Intelligence and Statistics (2003) 546431.

[3] Y. Guo, D. Schuurmans, Efficient global optimization for exponential family PCA and low-rank matrix factorization, In Proceedings of the 46th Annual Allerton Conference on Communication, Control, and Computing (2008) 1100-1107.

580

[4] I. T. Jolliffe, N. T. Trendafilov, M. Uddin, A modified principal component technique based on the lasso, Journal of Computational and Graphical Statistics 12 (3) (2003) 531-547. 
[5] H. Zou, T. Hastie, R. Tibshirani, Sparse principal component analysis, Journal of Computational and Graphical Statistics 15 (2) (2006) 265-286.

[15] M. Journee, Y. Nesterov, P. Richtarik, R. Sepulchre, Generalized power method for sparse principal component analysis, Journal of Machine Learning Research 11 (2010) 517-553. 
[23] T. I. H. Consortium, A haplotype map of the human genome, Nature 437

(2005) 1299-1320.

[16] K. Mardia, J. Kent, J. Bibby, Multivariate Analysis, Academic Press, New York, 1979.

[17] M. J. Wainwright, M. I. Jordan, Graphical models, exponential families, and variational inference, Foundations and Trends in Machine Learning 1 (2008) 1-305.

[18] S. Lee, J. Z. Huang, A coordinate descent MM algorithm for fast computation of sparse logistic PCA, Journal of Computational Statistics \& Data Analysis 62 (2013) 26-38.

[19] A. J. Landgraf, Y. Lee, Generalized principal component analysis: Projection of saturated model parameters, Technical Report No. 892, Department of Statistics, The Ohio State University, 2015.

[20] A. S. Georghiades, P. N. Belhumeur, From few to many: Illumination cone models for face recognition under variable lighting and pose, IEEE Transactions on Pattern Analysis and Machine Intelligence 23 (6) (2001) 643-660.

[21] D. Cai, X. He, J. Han, Document clustering using locality preserving indexing, IEEE Transactions on Knowledge and Data Engineering 17 (12) (2005) 1624-1637.

[22] X. Chen, D. Cai, Large scale spectral clustering with landmark-based representation, in: Twenty-Fifth Conference on Artificial Intelligence (AAAI'11), 2011. 


\section{Author Biographies}

- Meng Lu received the BS and MS degrees in Computer Science respectively from China University of Mining\&Technology, Beijing, China, in 2007 and 2010, and the MS degree in Computer Science from University of South Florida, Tampa, FL, in 2013. Currently, she is a Ph.D. student with the department of Electrical and Computer Engineering at Texas A\&M University, College Station, TX. Her current research interests include bioinformatics, statistical machine learning and probabilistic modeling.

- Jianhua Z. Huang received his B.S. degree in 1989 and M.S. degree in 1992, both in probability and statistics, from Beijing University, China; and his Ph.D. in statistics from University of California, Berkeley in 1997. He is currently Professor of Statistics at Texas A\&M University. His research interests include computational statistics, semi- and non-parametric statistical methods, statistical machine learning, and applied statistics. He is a fellow of ASA and IMS.

- Xiaoning Qian (S'01-M'07) received the Ph.D. degree in Electrical Engineering from Yale University, New Haven, CT, in 2005. Currently, he is an assistant professor with the Department of Electrical \& Computer Engineering, Texas A\&M University, College Station, TX. He also is a courtesy assistant professor in the Department of Computer Science \& Engineering and the Department of Pediatrics at the University of South Florida, Tampa FL, in which he spent four years before joining Texas A\&M. He was with the Bioinformatics Training Program at Texas A\&M University, sponsored by the National Cancer Institute (NCI). His current research interests include computational network biology, genomic signal processing, and biomedical image analysis. 